\title{
Cavitation-enhanced nonthermal ablation in deep brain targets: feasibility in a large animal model
}

\author{
Costas D. Arvanitis, PhD, ${ }^{1}$ Natalia Vykhodtseva, PhD, ${ }^{1}$ Ferenc Jolesz, MD, ${ }^{1}$ \\ Margaret Livingstone, $\mathrm{PhD}^{2}$ and Nathan McDannold, $\mathrm{PhD}^{1}$
} 1Department of Radiology, Brigham and Women's Hospital, Harvard Medical School; and 2Department of Neurobiology, Harvard
Medical School, Boston, Massachusetts

\begin{abstract}
OBJECTIVE Transcranial MRI-guided focused ultrasound (TcMRgFUS) is an emerging noninvasive alternative to surgery and radiosurgery that is undergoing testing for tumor ablation and functional neurosurgery. The method is currently limited to central brain targets due to skull heating and other factors. An alternative ablative approach combines very low intensity ultrasound bursts and an intravenously administered microbubble agent to locally destroy the vasculature. The objective of this work was to investigate whether it is feasible to use this approach at deep brain targets near the skull base in nonhuman primates.
\end{abstract}

METHODS In 4 rhesus macaques, targets near the skull base were ablated using a clinical TcMRgFUS system operating at $220 \mathrm{kHz}$. Low-duty-cycle ultrasound exposures (sonications) were applied for 5 minutes in conjunction with the ultrasound contrast agent Definity, which was administered as a bolus injection or continuous infusion. The acoustic power level was set to be near the inertial cavitation threshold, which was measured using passive monitoring of the acoustic emissions. The resulting tissue effects were investigated with MRI and with histological analysis performed 3 hours to 1 week after sonication.

RESULTS Thirteen targets were sonicated in regions next to the optic tract in the 4 animals. Inertial cavitation, indicated by broadband acoustic emissions, occurred at acoustic pressure amplitudes ranging from 340 to $540 \mathrm{kPa}$. MRI analysis suggested that the lesions had a central region containing red blood cell extravasations that was surrounded by edema. Blood-brain barrier disruption was observed on contrast-enhanced MRI in the lesions and in a surrounding region corresponding to the prefocal area of the FUS system. In histology, lesions consisting of tissue undergoing ischemic necrosis were found in all regions that were sonicated above the inertial cavitation threshold. Tissue damage in prefocal areas was found in several cases, suggesting that in those cases the sonication exceeded the inertial cavitation threshold in the beam path.

CONCLUSIONS It is feasible to use a clinical TcMRgFUS system to ablate skull base targets in nonhuman primates at time-averaged acoustic power levels at least 2 orders of magnitude below what is needed for thermal ablation with this device. The results point to the risks associated with the method if the exposure levels are not carefully controlled to avoid inertial cavitation in the acoustic beam path. If methods can be developed to provide this control, this nonthermal approach could greatly expand the use of TcMRgFUS for precisely targeted ablation to locations across the entire brain. http://thejns.org/doi/abs/10.3171/2015.4.JNS142862

KEY WORDS focused ultrasound; neurosurgery; ablation; acoustic cavitation; image-guided therapy; surgical technique

$\mathrm{T}$ TransCranial MRI-guided focused ultrasound (TcMRgFUS) is an emerging thermal ablation method that offers a noninvasive alternative to neurosurgery and radiosurgery. ${ }^{8,15,16,18}$ The method uses a hemispherical phased array transducer and MRI guidance to safely and precisely focus acoustic energy at deep brain targets. ${ }^{11}$ Due to high acoustic absorption in the skull and other factors related to transcranial ultrasound propaga- tion, ${ }^{33}$ TcMRgFUS thermal ablation currently can be applied only at central brain regions without overheating the skull.

A number of animal studies have shown that when the ultrasound exposures (sonications) are combined with a microbubble ultrasound contrast agent, ablation can be achieved at substantially lower exposure levels. ${ }^{4,6,9,22,26,30,31,36}$ We have been evaluating this method to ablate tissue in

ABBREVIATIONS BBB = blood-brain barrier; ETL = echo train length; FUS = focused ultrasound; LFB = Luxol fast blue; TcMRgFUS = transcranial MRI-guided FUS. SUBMITTED December 18, 2014. ACCEPTED April 2, 2015.

INCLUDE WHEN CITING Published online September 18, 2015; DOI: 10.3171/2015.4.JNS142862. 
the brain at low acoustic intensities to reduce skull heating. ${ }^{10,20,21,32}$ These studies have shown not only that lower power results in less bulk heating, but also that microbubble-ultrasound interactions can produce mechanically induced vascular damage that results in localized ischemic tissue necrosis. This damage is presumably associated with inertial cavitation, which is accompanied by high-velocity jets and shockwaves that are generated when microbubbles collapse. It is possible that other factors, such as vascular spasm and the creation of gas emboli when bubbles coalesce, are involved.

Therefore, this technique offers 2 potential advantages over thermo-ablative TcMRgFUS. The first is related to the significantly lower powers used (approximately 2 orders of magnitude) and the associated lower risks from skull heating, which restrict the treatment envelope of the currently available clinical system only to central brain regions. The second is related to the alleviation of challenges associated with using thermo-ablative FUS to treat targets near the bone (e.g., deep brain), where skull absorption in the far field prevents reaching lethal heating at the targeted tissue, or where heat diffusion might result in irreversible damage to adjacent sensitive structures (e.g., optic tract).

Despite the anticipated benefits of this method and promising results in small animal models, a number of issues need to be resolved before this alternative method of ultrasound ablation can progress to human trials. While this method can circumvent skull heating, it introduces new challenges. The interaction of a population of circulating microbubbles with an acoustic field in vivo is a stochastic process that depends on a number of factors, including microbubble size and type, vessel size, vascular density, and sonication parameters. New methods that take these factors into account for treatment planning and control are needed.

The potential risks of the procedure, particularly for damage along the ultrasound beam path when structures deep in the brain are targeted, also need to be explored and fully understood. These risks may be exacerbated by the design of currently available TcMRgFUS systems, which use a hemispherical transducer with a low acoustic frequency. Low ultrasound frequencies are used to reduce absorption and aberration induced by the skull, which also results in a larger effective range in which the beam can be steered electronically. Since acoustic absorption decreases with frequency, use of a lower frequency increases the risk of "hotspots" produced by reflections and standing waves inside the skull, particularly when focusing near the skull base. $^{24,28}$ At the same time, a low frequency reduces the threshold for inertial cavitation, and the "helmet" transducer design places large portions of brain in the acoustic beam path.

The only way to adequately evaluate all these factors concurrently is to use an animal model with a large enough brain that the beam path length is comparable to that which might be encountered in human patients. The purpose of this study was therefore to investigate this ablation method at deep brain structures in nonhuman primates using a clinical TcMRgFUS system. Our goal was to determine whether it is feasible to ablate targets near the skull base at exposure levels above the threshold for inertial cavitation without inducing significant damage along the ultrasound beam path. The TcMRgFUS system we used was a low-frequency clinical prototype operating at $220 \mathrm{kHz} .{ }^{34}$ During sonication, we recorded the acoustic emissions produced by the microbubbles to characterize the cavitation activity and fine tune the sonication parameters. After each sonication we evaluated the tissue effects at different brain structures - first with MRI and then, after euthanizing the animal, with histological analysis.

\section{Methods \\ Animals}

Experiments were performed in accordance with procedures approved by the Harvard Medical School Institutional Animal Care and Use Committee. Tests were performed in 4 adult male rhesus macaques (weight $7-13 \mathrm{~kg}$ ). Each animal was anesthetized with ketamine $(4 \mathrm{mg} / \mathrm{kg}$ / $\mathrm{hr}$ ) and dexmedetomidine (0.01-0.02 mg/kg/hr) and intubated. The head was shaved, and a catheter was placed in a leg vein. During the procedure the heart rate, blood oxygenation levels, and rectal temperature were monitored. Body temperature was maintained with a heated water blanket. Monkeys were housed, fed, watered, and provided with environmental enrichment according to US Department of Agriculture, Office of Laboratory Animal Welfare, and Association for Assessment and Accreditation of Laboratory Care regulations.

\section{Device}

The device used was the ExAblate 4000 low-frequency TcMRgFUS system (InSightec). It consists of a 30-cmdiameter, hemispherical 1024-element phased array transducer operating at $220 \mathrm{kHz}$ coupled with a 1024-channel driving system, a treatment planning workstation, and a water cooling/circulation/degassing system. The driving system allows for individual control of the phase and amplitude for each element in the phased array. The array is used for electronic beam steering of the focal point, and can be used for aberration correction that can compensate for the human skull. ${ }^{7}$ This correction was not used here. The FUS transducer was operated in a burst mode obtained via a gating signal provided by an arbitrary waveform generator (model 396, Fluke), which also triggered the acquisition for the system used to monitor acoustic emissions (see below). The system was integrated with a clinical 3-T MRI unit (GE Healthcare). Imaging was performed with a 14-cm-diameter receive-only surface coil (constructed in-house). To facilitate the experiments, the hemisphere transducer of the TcMRgFUS array faced upward (i.e., rotated $90^{\circ}$ from its normal use in patients) and was filled with degassed water like a bowl. The animal was placed supine on the MRI scanner table with its head tilted backward so that the top of the head was submerged in water (Fig. 1).

The focal half-width and length of the focal pressure distribution produced by the transducer in water were provided by the manufacturer and were approximately 4.2 and $9.0 \mathrm{~mm}$, respectively. Details on the procedure used to estimate the pressure amplitude are described elsewhere. ${ }^{2,17}$ Briefly, based on measurements with a calibrat- 


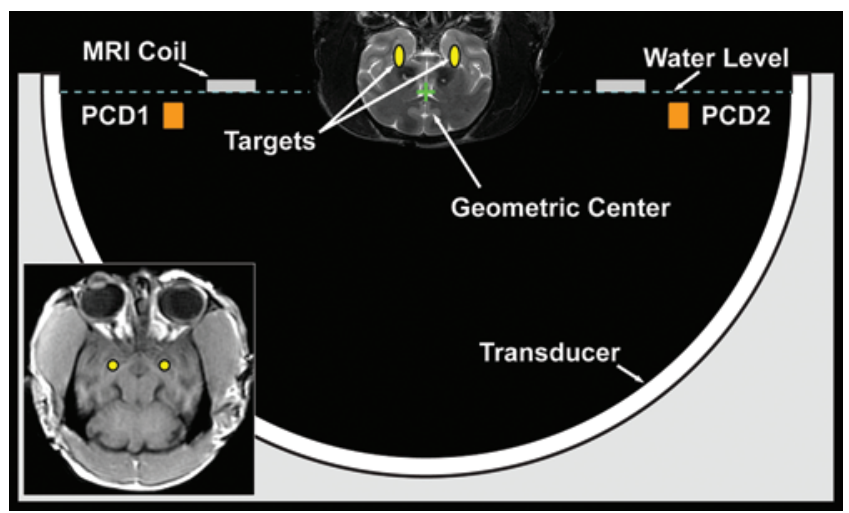

FIG. 1. Experimental setup. Coronal T2-weighted MR image superimposed on a diagram (drawn approximately to scale) of the 30 -cm-diameter hemispherical transducer of the clinical TcMRgFUS system. The locations of the 2 passive cavitation detectors (PCDs) used to monitor the acoustic emissions and the MRI surface coil are drawn. The locations of the geometric center of the transducer and the focal targets near the skull base are also indicated. Inset: Axial T1-weighted MR image used for selecting the 2 targets next to the optic tract in both hemispheres. The dimensions of the targets drawn in this figure correspond to the intensity focal half-width and length ( 4.2 and $9 \mathrm{~mm}$, respectively). Figure is available in color online only.

ed hydrophone (model TC4038-1, Reson Inc.) and an ex vivo macaque skull, we estimate that $1 \mathrm{~W}$ acoustic power corresponded to an estimated peak negative pressure amplitude of $223 \mathrm{kPa}$ in the brain at the geometrical focus. The skull base targets were approximately $2-3 \mathrm{~cm}$ away from the geometrical focus. This amount of electronic beam steering resulted in a decrease in focal pressure measured with a hydrophone in water of $10 \%-25 \%$. This reduction and other factors likely introduced uncertainty in these estimates.

\section{Acoustic Emission Monitoring and Analysis}

The acoustic emissions from the oscillating microbubbles offer characteristic signatures that allow for remote assessment of the type of oscillations and offer a potential way to guide this method. Strong harmonic and/or suband ultraharmonic acoustic emissions in the absence of broadband signal are indicative of stable volumetric oscillations. At higher pressure amplitudes, the microbubble oscillations become unstable and can collapse violently due to inertia of the surrounding medium. This collapse creates a pressure spike that is manifested in the frequency domain of the acoustic emissions as a broadband signal.

Passive cavitation detectors were used to record the acoustic emissions produced during the sonications. Two weakly focused (radius of curvature $=15 \mathrm{~cm}$ ), air-backed, rectangular $(4.0 \times 0.7 \mathrm{~cm})$ PZT transducers with a center frequency of approximately $610 \pm 20 \mathrm{kHz}$ were constructed in-house and mounted in the water on each side of the animal's head, approximately $10 \mathrm{~cm}$ from the geometrical focus of the hemispherical phased array (Fig. 1).

One detector was connected to a band-reject filter (125-390 kHz; model 3944, Krohn-Hite Corp.), which applied $40 \mathrm{~dB}$ amplification. The second was connected to a bandpass filter (250-1000 kHz; model EC6081 [VP2000], Reson Inc.), which also applied $20 \mathrm{~dB}$ amplification. The emissions were recorded at a $10-\mathrm{MHz}$ sample rate using a high-speed digitizing card (NI PXI-5124, National Instruments). The time signal, frequency spectra, and magnitude of the emissions at different frequencies were displayed in real-time during each sonication using software developed in-house in Matlab (Mathworks) and stored for later analysis. Spectra were calculated using a fast Fourier transform with a Hanning window. In the spectra, we calculated the signal strength for the first 3 harmonics $(440,660$, and 880 $\pm 0.25 \mathrm{kHz}$ ) and the first 2 ultraharmonics (330 and 550 $\pm 0.250 \mathrm{kHz}$ ). For broadband emissions, a frequency band around the resonance frequency of the cavitation detector $(610 \pm 5 \mathrm{kHz})$ was used. The following equation was used to calculate the relative integrated power spectral strength $(M)$ in each frequency band of the spectra $(S(f))$ :

$$
M=\int_{f_{1}}^{f_{2}} \log \left(\frac{S^{2}(f)}{S_{0}^{2}(f)}\right) d f
$$

where $S_{0}(f)$ is the spectra of the emissions obtained under similar conditions without injected microbubbles. ${ }^{2}$ The units of $M$ are $\mathrm{Np} \bullet \mathrm{Hz}$. The reported harmonic and ultraharmonic strengths are averages of 3 harmonics (2nd-4th) and 2 ultraharmonics $(3 / 2,5 / 2)$ of the acoustic frequency.

\section{Sonications}

Overall, 13 targets were sonicated in 4 monkeys (Table 1). They were near or adjacent to the optic tract and centered on the amygdala or putamen. In the first animal, 2 overlapping targets were sonicated in each hemisphere; otherwise, the targets did not overlap. All sonications consisted of 10-msec bursts applied at rate of $1 \mathrm{~Hz}$ for $5 \mathrm{~min}-$ utes. The sonications were combined with intravenous administration of the microbubble ultrasound contrast agent Definity (Lantheus Medical Imaging). For 9 locations, this agent was administered as a bolus injection at the start of each sonication $(20 \mu \mathrm{l} / \mathrm{kg}, 2$ times the dose used for imaging). In 4 locations, an infusion pump (Spectra Solaris EP, Medrad) was used to deliver microbubbles throughout the exposures. To keep the floating microbubbles mixed throughout the infusion, a length of plastic tubing (inner diameter $1 \mathrm{~mm}$ ) with a volume of $5 \mathrm{ml}$ was coiled, attached to a horizontal plate, and slowly filled with a solution of $20 \mu \mathrm{l} / \mathrm{kg}$ Definity diluted in $5 \mathrm{ml}$ PBS. The infusion pump pushed physiological saline through the tubing, thereby ensuring that the microbubbles were well mixed throughout the infusion. The infusion rate was $0.1 \mathrm{ml} / \mathrm{min}$ for the first 10 seconds and $0.02 \mathrm{ml} / \mathrm{min}$ thereafter.

In the first 2 sessions, we used our prior experience with this system ${ }^{2,17}$ to select an acoustic power level that was expected to be above or slightly below the inertial cavitation threshold to use during the ablation sonications. Thereafter, we measured the thresholds for stable and inertial cavitation at each sonicated target as shown before. ${ }^{2}$ In brief, the TcMRgFUS system was programmed to sonicate at increasing power levels with each burst; this power ramp was repeated 4 times at each location. Thresholds for harmonic, ultraharmonic, and broadband emissions were determined. The emissions signal was considered to be above the threshold when its magnitude was greater than 3 times the standard deviation of the noise floor. In 11 lo- 
TABLE 1. Experimental parameters and results

\begin{tabular}{|c|c|c|c|c|c|c|c|c|c|c|}
\hline \multirow[b]{2}{*}{$\begin{array}{c}\text { Animal } \\
\text { No. }\end{array}$} & \multirow[b]{2}{*}{ Location } & \multirow[b]{2}{*}{$\begin{array}{c}\text { Acoustic } \\
\text { Power (W) }\end{array}$} & \multirow[b]{2}{*}{$\begin{array}{l}\text { Pressure Amp } \\
\qquad(\mathrm{kPa})\end{array}$} & \multirow[b]{2}{*}{$\begin{array}{l}\text { Injection } \\
\text { Method }\end{array}$} & \multicolumn{2}{|c|}{ Lesion Size $(\mathrm{mm})$} & \multirow{2}{*}{$\begin{array}{c}\text { Duration Btwn } \\
\text { FUS \& Histological } \\
\text { Analysis }\end{array}$} & \multicolumn{3}{|c|}{ Acoustic Emission Threshold (kPa) } \\
\hline & & & & & Focal Plane & Beam Path ${ }^{*}$ & & Harmonics & Ultraharmonics & Broadband \\
\hline \multirow[t]{2}{*}{1} & $1-2$ & 4 & 444 & Bolus & $5 \times 5$ & $5 \times 6$ & $3 \mathrm{hrs}$ & & & \\
\hline & $3-4$ & 5 & 496 & Bolus & $6 \times 7$ & $7 \times 8$ & $3 \mathrm{hrs}$ & & & \\
\hline \multirow[t]{4}{*}{2} & 1 & 2 & 314 & Bolus & 0 & 0 & 7 days & & & \\
\hline & 2 & 3 & 385 & Bolus & 0 & 0 & 7 days & & & \\
\hline & $3 \dagger$ & 5 & 496 & Bolus & $3 \times 3$ & $9 \times 6$ & $4 \mathrm{hrs}$ & 210 & 420 & 440 \\
\hline & $4 \dagger$ & 5 & 496 & Bolus & $4 \times 4$ & $4 \times 8$ & $4 \mathrm{hrs}$ & 220 & 470 & 470 \\
\hline \multirow[t]{2}{*}{3} & 1 & 3.5 & 415 & Bolus & $4 \times 6$ & $9 \times 6$ & 3 days & 240 & 340 & 340 \\
\hline & 2 & 3.5 & 415 & Infusion & $4 \times 4$ & $6 \times 4$ & 3 days & & & \\
\hline \multirow[t]{3}{*}{$4 \ddagger$} & 1 & 6.4 & 561 & Infusion & $4 \times 3$ & $4 \times 4$ & 7 days & 260 & 490 & 510 \\
\hline & $2 \dagger$ & 6.4 & 561 & Infusion & $2 \times 1$ & $2 \times 2$ & $4 \mathrm{hrs}$ & 390 & 540 & 540 \\
\hline & $3 \dagger$ & 4.9 & 491 & Infusion & $2 \times 2$ & $3 \times 3$ & $4 \mathrm{hrs}$ & 390 & 540 & 540 \\
\hline
\end{tabular}

Amp = amplitude.

* Measured in the histology section that had the largest lesion diameter. If more than one area with damage was found, the dimensions of the entire affected region containing these areas is listed.

$\dagger$ Location sonicated in a second session 1 week after the first.

$\ddagger$ Sonicated in 1 hemisphere only due to a metallic pellet in the contralateral hemisphere that caused severe MRI artifacts.

cations, ablation was performed at power levels $4 \%-22 \%$ above the broadband emissions threshold. In 2 locations in the second animal the power was set purposely below this threshold.

\section{MRI}

The targets were selected using a 3D fast spoiled gradient echo sequence with inversion recovery preparation (TR $5.3 \mathrm{msec}$, TE $2.0 \mathrm{msec}$, TI $600 \mathrm{msec}$, FA 10 ${ }^{\circ}$, FOV 12 $\mathrm{cm}$, matrix $128 \times 128$, slice thickness $2 \mathrm{~mm}$ ). After sonication, 3D $\mathrm{T} 2 *$-weighted spoiled gradient echo images (TR $33 \mathrm{msec}, 19 \mathrm{msec}$, echo train length [ETL] 1, FA 15', FOV $12 \mathrm{~cm}$, matrix $256 \times 256$, slice thickness $1 \mathrm{~mm}$ ) were acquired to visualize hypointense areas produced by red blood cell extravasations resulting from inertial cavitation and vascular damage. ${ }^{12}$ T2-weighted fast spin echo (FSE) images (TR $4500 \mathrm{msec}$, TE $85.8 \mathrm{msec}$, echo train length, ETL 8, FOV $12 \mathrm{~cm}$, matrix $256 \times 256$, slice thickness 3 $\mathrm{mm}$ ) were acquired to further visualize the lesions and the surrounding edema. T1-weighted FSE images (TR 500 msec, TE $14 \mathrm{msec}$, ETL 4, FOV $12 \mathrm{~cm}$, matrix $256 \times 256$, slice thickness $3 \mathrm{~mm}$ ) acquired before and after intravenous administration of the MRI contrast agent Gd-DTPA (0.1 mmol/kg; Magnevist, Berlex Laboratories, Inc.) to identify regions of blood-brain barrier (BBB) disruption.

\section{Histology}

The animals were euthanized for histological examination at approximately 3 hours, 72 hours, and 1 week after the last sonication session. The animals were deeply anesthetized with ketamine $(15 \mathrm{mg} / \mathrm{kg}$ intramuscularly) and then killed with an overdose of pentobarbital $(100 \mathrm{mg} /$ $\mathrm{kg}$ ). They were then perfused transcardially with $1 \mathrm{~L} 0.9 \%$ $\mathrm{NaCl}$, followed by $2 \mathrm{~L} 10 \%$ buffered formalin phosphate. The brains were removed and placed in $10 \%$ buffered formalin phosphate for immersion fixation. Then they were cut into 2-mm blocks and macrophotographs of both sides of each block were obtained. The blocks were then em- bedded in paraffin and serially sectioned at $5 \mu \mathrm{m}$. Every 40th section was stained with $\mathrm{H} \& \mathrm{E}$ and Luxol fast blue (LFB). Additional sections were stained with Nissl (for neurons and glia cells) and Bielschowsky's silver stain (for nerve fibers).

\section{Results \\ Acoustic Emissions}

An example plot of the strength of the acoustic emissions as a function of the peak negative pressure is shown in Fig. 2. Strong harmonic emissions appeared at relatively low-pressure amplitudes, whereas broadband, ultraharmonic and subharmonic emissions appeared simultaneously at higher amplitudes (Fig. 2A). The strength of the harmonic emissions increased linearly with the peak negative pressure. Spectrogram analysis (Fig. 2B) revealed that the strength of emissions sometimes appeared in the middle of the 10 -msec bursts when the pressure amplitude was near the threshold; the emissions were sustained throughout the bursts at higher amplitudes. The thresholds for harmonic, ultraharmonic, and broadband emissions varied among the different animals (Table 1).

Example plots of the strength of the acoustic emissions as a function of time during ablation are shown in Fig. 3. In these examples from Animal 3, very strong broadband emissions of $5 \mathrm{~Np} \cdot \mathrm{Hz}$ and above were observed during the first 6 seconds of the sonication. This signal strength then decreased, and low-level broadband emissions $(1-3 \mathrm{~Np} \cdot \mathrm{Hz})$ were observed throughout the rest of the sonication. With an infusion, strong broadband emissions were observed sporadically throughout the sonication, and the strength of the low-level broadband signal increased over time. In 2 locations in Animal 2 where the microbubbles were administered as a bolus, the behavior was similar: strong broadband signals at the start of sonication for about 10 seconds and then low-level broadband activity throughout the rest of the sonication. In the other 6 sonications ( 4 with bolus, 

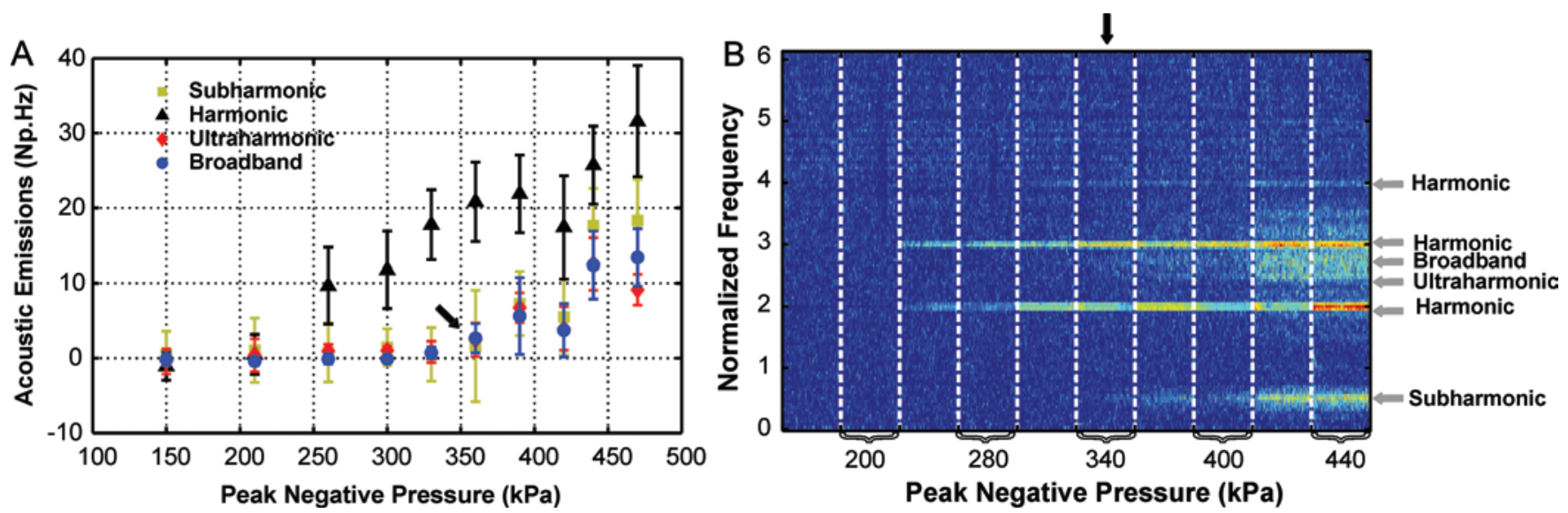

FIG. 2. Animal 3. Acoustic emissions at different frequency bands as a function of the peak negative pressure. A: Representative acoustic emissions during sonications showing harmonic, subharmonic, ultraharmonic, and broadband emissions at increasing pressure amplitudes. Each point is the average of 4 bursts $( \pm$ SD). Harmonic emissions appeared first at $260 \mathrm{kPa}$, whereas the broadband emissions appeared at $360 \mathrm{kPa}$. Harmonic emission increased linearly with pressure. B: Spectrograms showing the acoustic emissions during the 10-msec bursts at increasing acoustic pressure amplitudes. For this analysis, each 10-msec burst was segmented into 100 segments that were $100 \mu \mathrm{sec}$ long. Near the threshold for the appearance of each spectral component, signals appeared in the middle of the $10-\mathrm{msec}$ burst. At higher values it was almost constant over the entire burst. Broadband emissions were detected in a region centered on the resonant frequency of the passive cavitation detector (610 kHz). The black arrow indicates the pressure threshold for the onset of broadband emissions (inertial cavitation threshold). The data shown in $\mathrm{A}$ and $B$ are from different experiments. Figure is available in color online only.
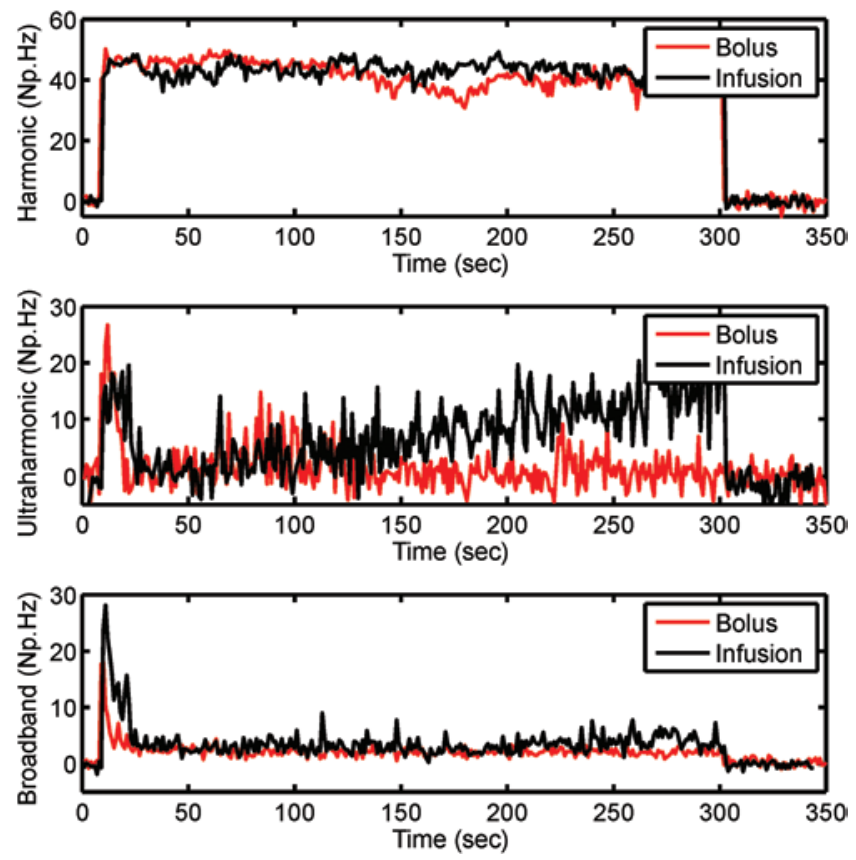

FIG. 3. Animal 3. Example plots showing the strength of the acoustic emissions in different frequency bands as a function of time for 2 locations. These two locations were in the same structure in each hemisphere and were sonicated at the same exposure level. In one hemisphere, the microbubbles were administered as a bolus; in the other they were administered as an infusion. The increase in emissions due to the arrival of the microbubbles at about 10 seconds is evident. Strong harmonic and ultraharmonic emissions were recorded during sonications of both targets. Strong broadband emissions were observed at the beginning of the sonication. For the remainder of the sonication, the strength of the broadband signals was greatly reduced. With an infusion, strong broadband signal spikes during individual bursts were observed sporadically during this period. Figure is available in color online only.
2 with infusion), strong broadband emissions of $5 \mathrm{~Np} \cdot \mathrm{Hz}$ or above were observed throughout the sonication. The dynamics of the ultraharmonic emission mirrored that of the broadband emission. In every case, the harmonic emissions were strong throughout the entire sonication.

\section{MRI Findings}

The lesions were clearly evident on $\mathrm{T} 2 *$-weighted imaging, where they appeared as hypointense regions of similar size or smaller than the focal region of the TcMRgFUS device (Fig. 4A). Comparison with presonication imaging revealed no MRI-evident changes in the beam path in the near- or far-field in this imaging. On T2-weighted imaging (Fig. 4B and C), a hyperintense rim surrounding the lesion was observed. On contrast-enhanced T1-weighted imaging, BBB disruption was evident in a region surrounding the lesion in the focal plane (Fig. 4D) and in a roughly cone-shaped region in the prefocal area of the beam path (Fig. 4E). This BBB disruption extended 6-19 mm below the focal plane. Hyperintense regions were also observed in this region in T2-weighted imaging. However, since the T2-weighted imaging was acquired after MRI contrast administration, it was not clear if the hyperintense signal was due to edema or to contrast extravasation across the BBB.

\section{Histological Findings}

Except for the 2 targets in Animal 2 that were sonicated below the inertial cavitation threshold, discrete lesions were found in all the targeted locations. Histological examination revealed that microbubble-enhanced sonications resulted in vascular damage throughout the lesion. Evaluation at different times after sonication revealed tissue undergoing ischemic necrosis. The lesions in the 2 animals euthanized 3 hours after treatment had red blood cell extravasations from the damaged capillaries. Ische- 

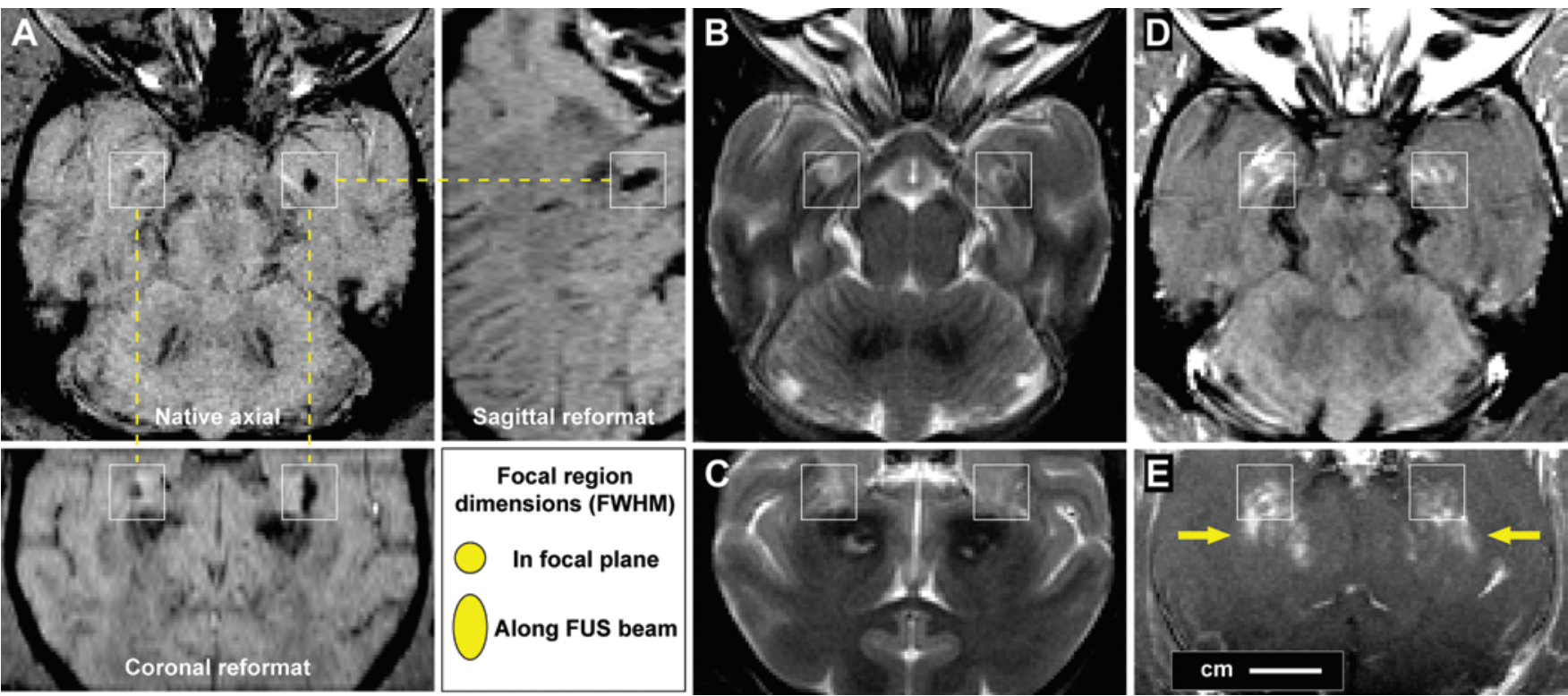

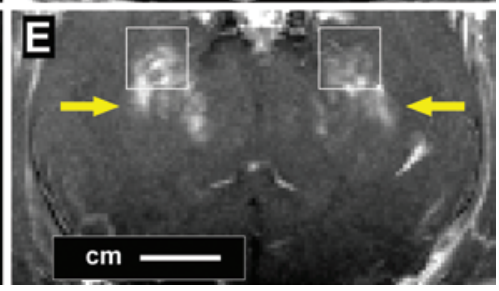

FIG. 4. Animal 3. MR images acquired immediately after bilateral ablation next to the optic tract. A: On the T2*-weighted MR images, the lesions appeared as well-defined hypointense regions in this case. Both the native axial imaging and coronal and sagittal reformats are shown. Comparison with imaging acquired before sonication detected no MRI-visible effects outside of these hypointense areas. The size of the full-width, half-maximum (FWHM) pressure distribution of the focus is shown for comparison. B and C: Axial and coronal T2-weighted MR images showing the lesions surrounded by a hyperintense rim, presumably from edema. D and E: Axial and coronal contrast-enhanced T1-weighted images showing areas of BBB disruption surrounding the lesion and in a prefocal region more dorsal to the focal plane. To show the extent of the BBB disruption, which occurred over several planes, a maximum intensity projection of five adjacent images is shown (E). Figure is available in color online only.

mic necrosis, presumably due to damage to the blood supply in the targeted locations, was also evident (Fig. 5A-C). The lesions had a patchy appearance; light-stained necrotic patches were intermixed with more deeply stained "preserved" areas, presumably due to damaged but not yet destroyed blood flow in the capillary blood vessels. These surviving "preserved" neurons clearly contrasted with dead and dying cells. Even though the lesions partly overlapped the optic tract, the optic tract fibers appeared intact, and the gross damage observed in the neighboring putamen or amygdala was not observed. In some areas in the interface between the optic tract and the lesions, there was a partial separation of the meningeal sheaths and damage to the small meningeal blood vessels accompanied by red blood cell extravasations. The optic tract nerve fibers within these small hemorrhagic areas appeared disarranged and rarefied (Fig. 5D and E). The nerve fibers in the rest of the optic tract were intact (Fig. 5F).

Liquefactive necrosis was evident throughout the lesions in the animal euthanized 3 days after sonication. There was a total loss of tissue architecture and formation of semiliquid mass of dead cells (Fig. 6A-C). More extensive extravasations were observed in this animal when Definity was administered as an infusion than when a bolus was used (Fig. 6D and E). The marginal area of the infarcted territory contained numerous macrophages engulfing degenerate material (Fig. 6F). The necrotic tissues were mostly removed at 1 week after sonication, and large numbers of macrophages and activated astrocytic glial cells filled the infarcted territory, indicating the processes of organization and reparation (gliosis) (Fig. 7). Vascular endothelial proliferation was seen at the edge of the infarct.
In several cases, it was clear that the damage extended outside the focal region, and tissue damage was found in surrounding areas where BBB disruption was observed in MRI. This damage was most prevalent in the prefocal region dorsal to the focal plane (i.e., toward the cap of the hemispherical transducer). The dimensions of the damaged area in this region were larger than those of the lesion in the focal plane. In several cases, this damage appeared as 2 or more relatively distinct areas. An example of prefocal damage is shown in Fig. 8A and B. The dimensions of the damaged area in the prefocal area and those for the lesion in the focal plane are listed in Table 1. When more than one area of damage was observed in the focal region, the dimensions of the total affected area are listed in the table. In addition to the damage in the prefocal region, at depths near the focal plane, damage was observed 1-2 $\mathrm{mm}$ from the lesion boundary in some cases, particularly at the interface between the optic tract and the adjacent gray matter in the putamen (Fig. 8C and D).

\section{Discussion}

This study demonstrates the feasibility of producing lesions deep in the brain using nonthermal ablation, a method where intravenously injected microbubbles are used to enable destruction of the microvasculature at exposure levels that do not produce bulk heating. The time-averaged acoustic power needed to produce these lesions $-64 \mathrm{~mW}$ or less-was less than 2 orders of magnitude of that needed for thermal ablation with this device without the injection of microbubbles. ${ }^{34}$ With this reduction in acoustic power, the risk of overheating the skull is largely avoided, which could expand the targetable areas in the brain with this de- 


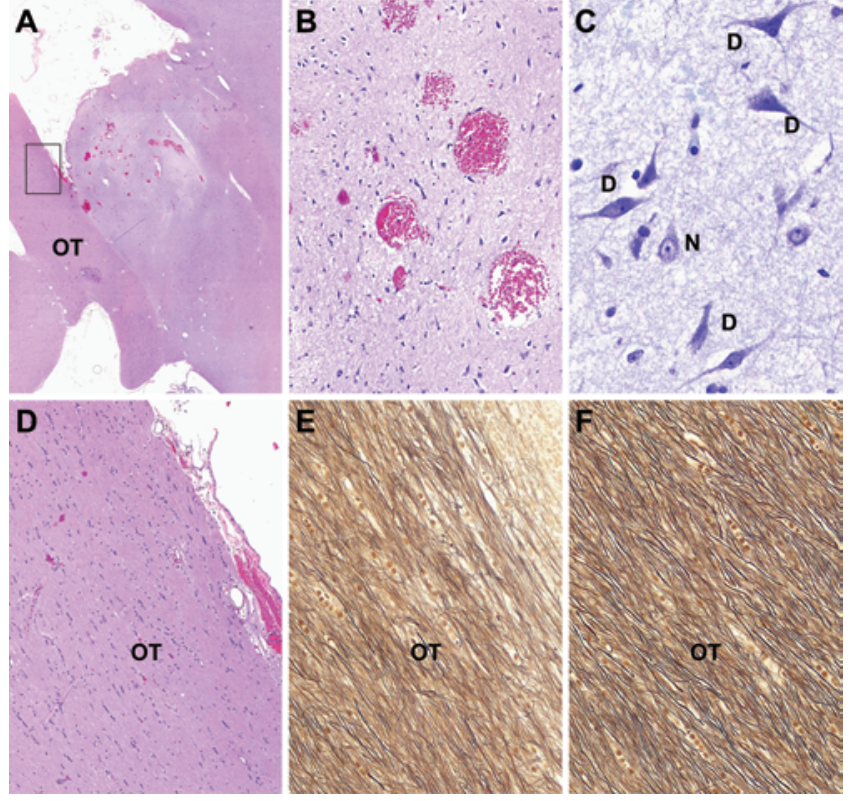

FIG. 5. Animal 1. Histological appearance of a lesion 3 hours after sonication. In this case, 2 overlapping targets were sonicated, and in 1 sonication the focal area overlapped the optic tract (OT). A: The lesion was characterized by the disruption of the capillary vessels with extravasations of red blood cells. B: Ischemic necrosis in the treated areas due to termination of blood supply. C: Dead shrunken neurons with pyknotic nuclei (D) contrasting with normal neurons (N). D: A partial separation of the optic tract meningeal sheath and intermeningeal hemorrhages (rectangle in A). E: Disarranged and rarefied nerve fibers within hemorrhagic boundary area in the same area. $F$ : The nerve fibers in the rest of the optic tract appeared intact. H\&E (A, B, and D); LFB (C); Bielschowsky's silver stain (E and F); original magnification $\times 1.25(A) ; \times 20(B) ; \times 100(C)$; $\times 10(D) ; \times 40(E$ and $F)$. Figure is available in color online only.

vice and increase the number of patients who can benefit from this less invasive alternative to resection.

The lesions appeared to be created via ischemia, consistent with earlier tests on this method in small animals..$^{10,20,21,32}$ Presumably, the ischemia was created by mechanical damage to the microvasculature induced by inertial cavitation. The role of inertial cavitation was evident from the lack of lesions produced in the experiment where the sonications were applied below the threshold for broadband emissions, and from histological examination, which found extravasated erythrocytes presumably caused by microvasculature damage when the pressure amplitude was above the inertial cavitation threshold. It is possible that other mechanisms, such as vascular spasm or the trapping or coalescence of microbubble fragments could be involved.

Necrosis produced with this microbubble-enhanced approach may not be immediate and appears to take more time to develop than with high-temperature thermal ablation, where cell coagulation can be immediate. Some preserved regions were evident within the lesion boundary 3 hours after sonication, but at later times no surviving cells were found within the lesion. In contrast, we anticipate that the ablated tissue will be removed more rapidly with microbubble-enhanced ablation than with thermal ablation, where thermally coagulated tissue can remain for extended periods. ${ }^{3,5}$ One week after sonication, the necrotic
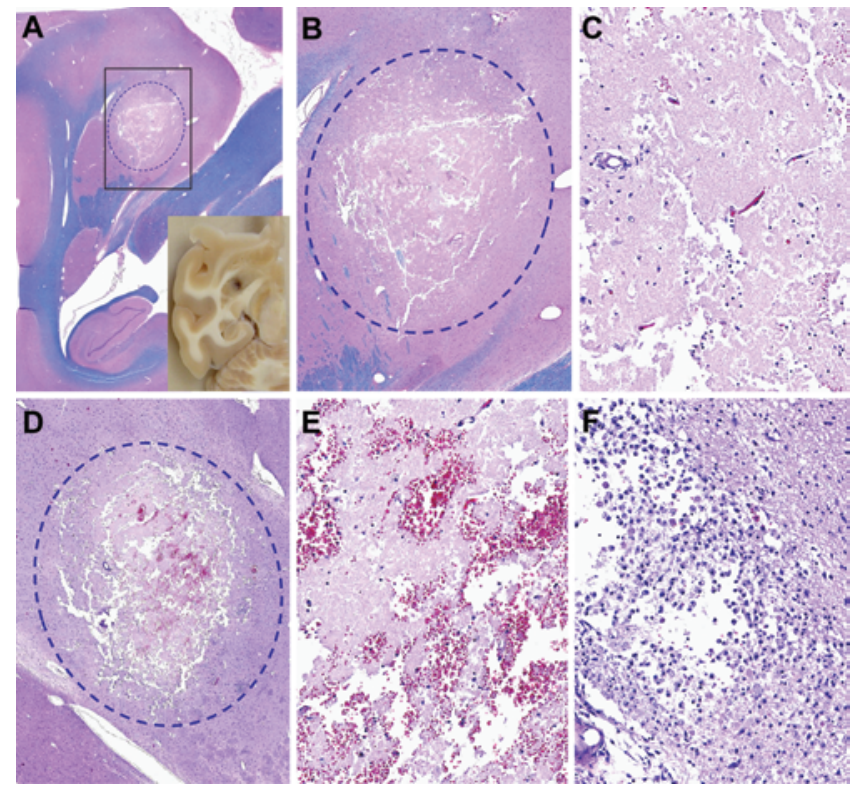

FIG. 6. Animal 3. Appearance in H \& E-stained sections of the 2 lesions in the amygdala 3 days after sonication. A-C: Colliquative necrosis with a total loss of tissue architecture and formation of a semiliquid mass of dead cells. In this example, the microbubbles were administered as a bolus injection. Examination of the tissue blocks found hemorrhagic lesions adjacent to the optic tracts (inset in A). D and E: More extensive hemorrhages in the location sonicated in the contralateral hemisphere. Here the microbubbles were infused slowly over the 5 min sonication. F: Numerous macrophages engulfing degenerated material at the margins of the infarcted territory. Original magnification $\times 0.625(\mathrm{~A}) ; \times 2.5$ $(B) ; \times 10(C) ; \times 2.5(D) ; \times 20(E$ and $F)$. Figure is available in color online only.

tissue was largely removed and the lesion was infiltrated by macrophages. Prior tests in rats suggest that after a few weeks the damaged area will be resorbed and in some cases a fluid-filled cyst-like region remains.

The tissue effects appeared to follow the beam profile of the device. In the focal plane, congruent lesions of ischemic necrosis that were smaller than the $50 \%$ contours of acoustic pressure of the TcMRgFUS device were found. Some effects, however, were observed outside this area. BBB disruption was observed in a fairly large area surrounding the focal lesions; in the worst cases it extended $10 \mathrm{~mm}$ or more away from the lesion. This finding is not surprising, since, except for the exposure level, the sonications had the same parameters as those used previously for BBB disruption with this device. ${ }^{17}$ This BBB disruption in the surrounding tissue likely coincided with areas that exceed a certain pressure threshold, which, to some extent, is probably unavoidable. However, ultrasound-induced BBB disruption is unlikely to result in tissue damage ${ }^{13}$ and thus should not be considered a significant impediment to the use of this method. In fact, for example, in tumor ablation it might be useful, as the method could be accompanied by the administration of chemotherapy or other agent for local treatment in the surrounding margins. ${ }^{29}$ Such a vascular strategy will restrict the supply of nutrients and oxygen in tumor, and, thus suppress its growth. Therefore, we envision that this work may lead to the development of new therapeutic strategies to treat solid tumors using 

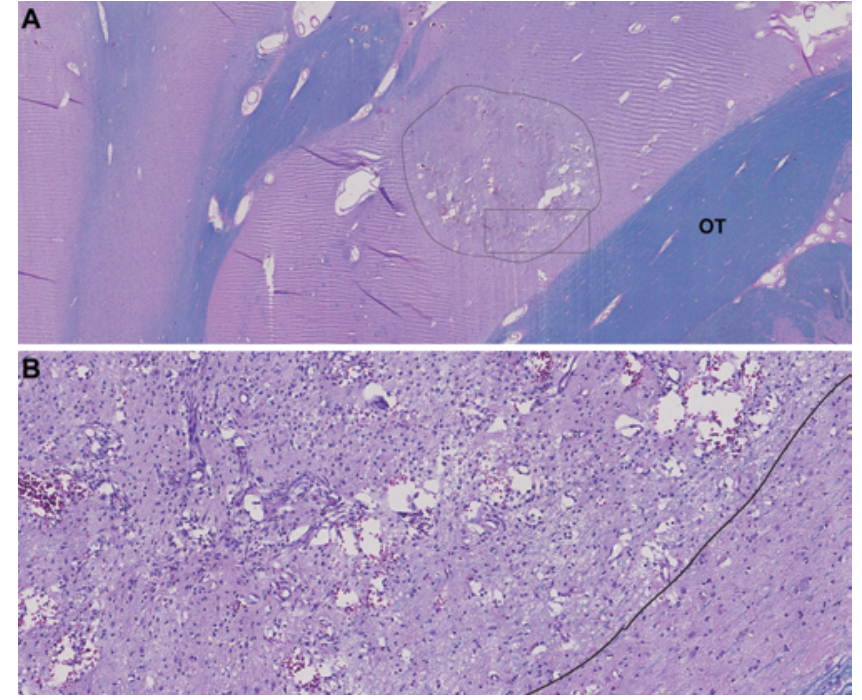

FIG. 7. Monkey 4. Appearance in an H \& E/LFB-stained section of a lesion close to the optic tract (OT) 1 week after sonication. A: A welldefined lesion was found consisting of necrotic tissue that was infiltrated by macrophages, activated astrocytic glial cells, and proliferating blood vessels. B: Magnification of box in A showing the lesion boundary. Original magnification $\times 1.25(\mathrm{~A}) ; \times 10(\mathrm{~B})$. Figure is available in color online only.

acoustic cavitation to interrupt the blood supply to the tumor core, while delivering therapeutic agents to surrounding margins.

In addition to the BBB disruption, in several cases damage in the ultrasound beam path was produced, particularly in the prefocal region, as shown in Fig. 8A and B. This result was evident in histology, where the dimensions of the damaged areas in these prefocal areas were larger than those in the focal plane. This damage was presumably due to exceeding the inertial cavitation threshold in those areas during the 5-minute sonications. Since the focal region was close to the skull base, reflections and standing waves might also have contributed. ${ }^{24,28}$ A Definity dose 2 times the suggested dose for ultrasound imaging and shielding of the ultrasound beam due acoustic attenuation resulting from a high microbubble concentration in the prefocal region might have also contributed. This finding suggests that the exposure level should be tailored to be close to the inertial cavitation threshold and demonstrates the need for improved monitoring and control over the procedure. Acoustic emissions monitoring, as was used here, and transcranial passive acoustic mapping ${ }^{1,23}$ are promising techniques to provide this control.

The location of the targets also likely contributed to the unwanted effects in the beam path. The targets at the skull base required steering the focal point above the water level, which resulted in $2-3 \mathrm{~cm}$ of the upper portion of the transducer to be clipped. This clipping effectively reduced the focusing gain of the transducer, which elongated the focal region, and, due to irregular clipping, probably made the acoustic field asymmetrical. It also partially blocked the acoustic path to the passive cavitation detectors, which probably reduced their sensitivity to acoustic emissions at the focal point. It is possible that the detectors' maximum sensitivity was in the prefocal region and we were
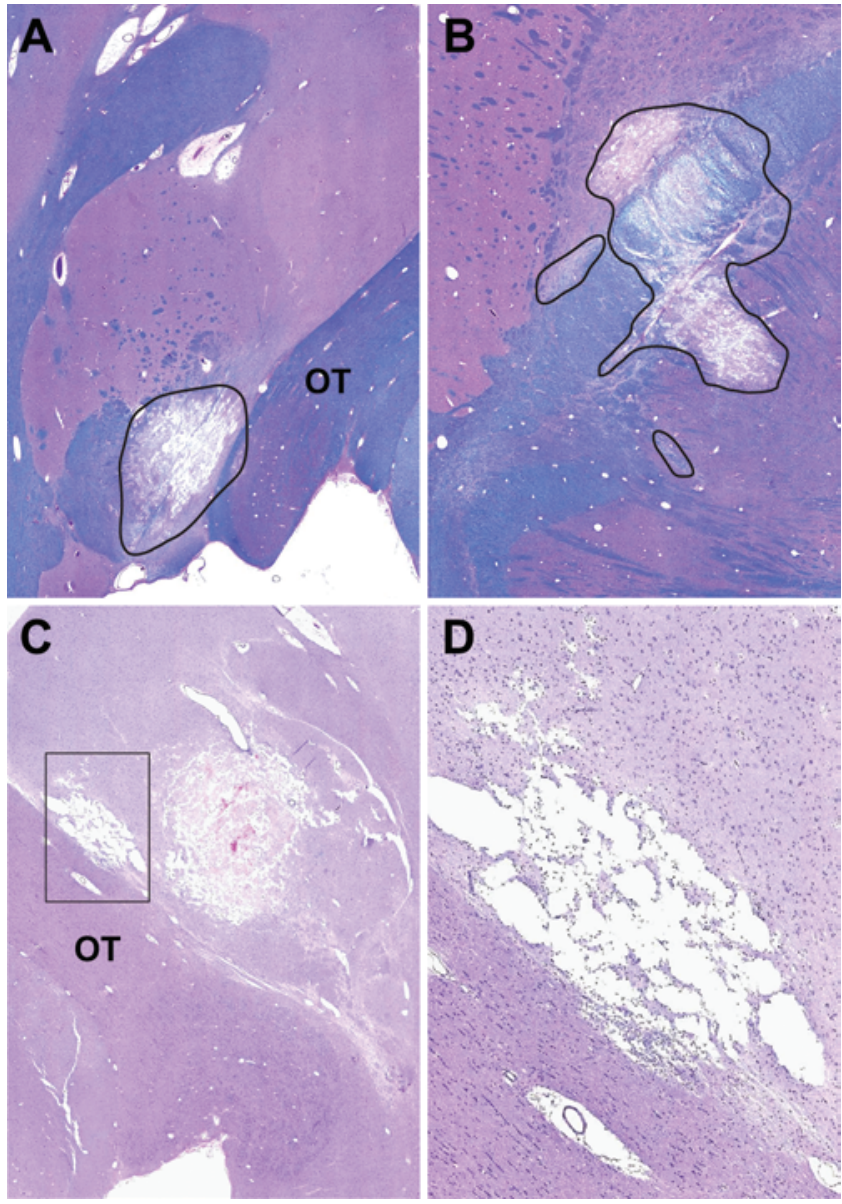

FIG. 8. Histology showing damage outside of the focal lesion. A and B: Animal 2. $\mathrm{H} \&$ E/LFB-stained sections showing the damage in the focal plane and in the prefocal region 4 hours after sonication. The lesion (outlined, dimensions: $\sim 3.2 \times 2.6 \mathrm{~mm}$ ) overlapping the optic tract (OT) from a section near the focal plane $(A)$. A section from a prefocal region approximately $5 \mathrm{~mm}$ closer to the transducer cap (B). Here the main lesioned area was larger $(\sim 4.1 \times 5.8 \mathrm{~mm})$ and more irregularly shaped. Two tiny areas with satellite damage were also found. C and D: Animal 3. H \& E-stained section near the focal obtained 3 days after sonication, showing damage approximately $1.4 \mathrm{~mm}$ outside of the lesion at the optic tract boundary. Original magnification $\times 1.25(A-C) ; \times 5(D)$. Figure is available in color online only.

setting the exposure level based largely on cavitation activity there. Furthermore, skull aberration may have also played a role. While aberration of the longitudinal beam is not likely to be significant at such a low frequency, ${ }^{35}$ mode conversion to shear waves likely occurred where portions of the transducer had large angles of incidence. ${ }^{33}$ At the deep, lateral targets investigated here, a wide dispersion of angles between the skull and the face of the transducer occurred, which may have resulted in more aberration than would occur at centrally located targets. The use of aberration correction that takes into account such mode conversion $^{7}$ may improve the focusing and reduce the risk of damage outside the focal region. Better treatment planning methods to reduce reflections are also needed, particularly when sonicating targets close to the skull.

The pattern of damage also appeared to vary for different tissue structures, in that gray matter was more sus- 
ceptible to damage than were fiber tracts. Presumably, this was due largely to variations in vascular density and microbubble concentrations, but other factors such as blood vessel diameter might also play a role. The threshold for inertial cavitation might be lower in large blood vessels, ${ }^{27}$ and vessels with a thicker wall may be able to recover after inertial cavitation. This variability among different tissue structures was most evident when comparing the effects in highly vascular gray matter, where contiguous lesions were produced, to that of the optic tract, which has a lower vascular density and where the damage was substantially less. The boundary between the optic tract and the adjacent gray matter was revealed a clear differential sensitivity to ultrasound/microbubble effects (Fig. 8B and C). It is interesting that this boundary area appeared sensitive to damage, as others have shown that such areas have a lower threshold to nucleate cavitation.

Despite the observed effects outside of the focal region the presented data suggest that the ischemic penumbra is indeed very sharp and could potentially be sharper than thermo-ablative or ionizing radiation methods. However more work is needed, particularly in assessing the observed prefocal damage, before we can conclude on the size of the ischemic penumbra of this technique. Furthermore, a side-by-side comparison of the area that can be treated between this method and thermo-ablative FUS is essential. We are currently developing numerical simulations to perform this comparison in silico. The simulations will also allow us to study the formation of standing waves and identify the optimal frequency for this procedure. Additional experiments will be used to validate the numerical simulations, which will then be used to study the parameter space and develop treatment-planning tools. In addition, MRI to monitor the evolution of the lesion and provide the means to assess the procedure noninvasively is essential if this technique is to be used in the clinic.

The sonications were monitored with passive cavitation detectors. In each location, strong broadband signal was detected along with harmonic and ultraharmonic emission. These emissions were present throughout the 10 -msec bursts, indicating that the bubbles were not being destroyed at the beginning of the burst. In 2 animals, strong broadband signals were present only for a short time at the beginning of the sonication, which would be consistent with an interruption in blood flow in the focal region or the destruction of the microvasculature. In the other 2 animals, the broadband signals remained strong throughout the entire 5-minute sonication. It is not clear how this could happen if the blood vessels were being destroyed. Perhaps in those cases the inertial cavitation threshold was exceeded in a large blood vessel where the blood flow remained throughout the sonication. The behavior of inertially cavitating microbubbles in the vasculature is complex and more work is needed to understand how to interpret the emissions.

This was a feasibility study with several limitations. The number of lesions created was small and we did not examine the animals for functional changes. As described above, our passive cavitation detectors were not mounted optimally, which may have reduced their sensitivity and artificially increased our measured acoustic emissions thresholds.
While we were successful in creating deep lesions at low exposure levels, as we describe above, some damage occurred in the acoustic beam path in several cases. We expect that with finer control over the exposure level to drive the sonications at or just above the inertial cavitation that we can reliably restrict the damage to the focal region.

The submillimeter $(\sim 0.7 \mathrm{~mm})$ targeting accuracy that is possible with clinical TcMRgFUS systems, ${ }^{11}$ along with the tight focal region and clear demarcation of the lesions presented here, suggest that this technique, when optimized, may compare favorably to radiosurgery or other ablation techniques. Before ablation, one can use low-intensity sonication and MR temperature imaging to detect subthreshold heating ${ }^{14}$ or MR acoustic radiation force imaging to detect small tissue displacements ${ }^{19,25}$ and ensure that the focal point is correctly targeted within the resolution of MRI. Transcranial passive acoustic mapping can be used with microbubble-enhanced ablation to monitor the ablation in real time at a similar resolution. ${ }^{1}$ With these imaging methods we expect that TcMRgFUS can ultimately equal or exceed the precision of even proton beam therapy. Unlike with radiation, tissue effects induced by TcMRgFUS are also evident immediately in MRI, providing further assurance that the targeted tissue received a sufficient treatment.

\section{Conclusions}

This study demonstrates that when sonications are combined with an ultrasound contrast agent, this low frequency TcMRgFUS system can be used to ablate vascular brain targets deep in the brain in a primate model at very low exposure levels. Consistent with prior work in small animals, ${ }^{10,20,21,32}$ the sonications resulted in localized ischemic necrosis, presumably resulting from mechanically induced damage to the microvasculature. This is a promising method to expand the area within the brain that can be treated where focused ultrasound neurosurgery can be used. We also envision the development of novel methods to treat solid tumors in the brain. However, more work is needed to control the procedure to ensure that the desired effects only occur at the focal region.

\section{Acknowledgments}

This work was funded by a grant from the Focused Ultrasound Surgery Foundation. Additional support was supplied by NIH Grant P01CA174645. C. D. Arvanitis received support from R25CA089017, K99EB016971.

\section{References}

1. Arvanitis CD, Livingstone MS, McDannold N: Combined ultrasound and MR imaging to guide focused ultrasound therapies in the brain. Phys Med Biol 58:4749-4761, 2013

2. Arvanitis CD, Livingstone MS, Vykhodtseva N, McDannold $\mathrm{N}$ : Controlled ultrasound-induced blood-brain barrier disruption using passive acoustic emissions monitoring. PLoS One 7:e45783, 2012

3. Astrom KE, Belle, Ballantine HT Jr, Heidensleben E: An experimental neuropathological study of the effects of highfrequency focused ultrasound on the brain of the cat. J Neuropathol Exp Neurol 20:484-520, 1961

4. Burke CW, Klibanov AL, Sheehan JP, Price RJ: Inhibition of glioma growth by microbubble activation in a subcutaneous model using low duty cycle ultrasound without significant heating. J Neurosurg 114:1654-1661, 2011 
5. Carpentier A, Chauvet D, Reina V, Beccaria K, Leclerq D, McNichols RJ, et al: MR-guided laser-induced thermal therapy (LITT) for recurrent glioblastomas. Lasers Surg Med 44:361-368, 2012

6. Chung DJ, Cho SH, Lee JM, Hahn ST: Effect of microbubble contrast agent during high intensity focused ultrasound ablation on rabbit liver in vivo. Eur J Radiol 81:e519-e523, 2012

7. Clement GT, Hynynen K: A non-invasive method for focusing ultrasound through the human skull. Phys Med Biol 47:1219-1236, 2002

8. Elias WJ, Huss D, Voss T, Loomba J, Khaled M, Zadicario E, et al: A pilot study of focused ultrasound thalamotomy for essential tremor. N Engl J Med 369:640-648, 2013

9. Fujishiro S, Mitsumori M, Nishimura Y, Okuno Y, Nagata Y, Hiraoka M, et al: Increased heating efficiency of hyperthermia using an ultrasound contrast agent: a phantom study. Int J Hyperthermia 14:495-502, 1998

10. Huang Y, Vykhodtseva NI, Hynynen K: Creating brain lesions with low-intensity focused ultrasound with microbubbles: a rat study at half a megahertz. Ultrasound Med Biol 39:1420-1428, 2013

11. Hynynen K, Clement GT, McDannold N, Vykhodtseva N, King R, White PJ, et al: 500-element ultrasound phased array system for noninvasive focal surgery of the brain: a preliminary rabbit study with ex vivo human skulls. Magn Reson Med 52:100-107, 2004

12. Hynynen K, McDannold N, Vykhodtseva N, Jolesz FA: Noninvasive MR imaging-guided focal opening of the bloodbrain barrier in rabbits. Radiology 220:640-646, 2001

13. Hynynen K, McDannold N, Vykhodtseva N, Raymond S, Weissleder R, Jolesz FA, et al: Focal disruption of the bloodbrain barrier due to $260-\mathrm{kHz}$ ultrasound bursts: a method for molecular imaging and targeted drug delivery. J Neurosurg 105:445-454, 2006

14. Hynynen K, Vykhodtseva NI, Chung AH, Sorrentino V, Colucci V, Jolesz FA: Thermal effects of focused ultrasound on the brain: determination with MR imaging. Radiology 204:247-253, 1997

15. Lipsman N, Schwartz ML, Huang Y, Lee L, Sankar T, Chapman M, et al: MR-guided focused ultrasound thalamotomy for essential tremor: a proof-of-concept study. Lancet Neurol 12:462-468, 2013

16. Martin E, Jeanmonod D, Morel A, Zadicario E, Werner B: High-intensity focused ultrasound for noninvasive functional neurosurgery. Ann Neurol 66:858-861, 2009

17. McDannold N, Arvanitis CD, Vykhodtseva N, Livingstone MS: Temporary disruption of the blood-brain barrier by use of ultrasound and microbubbles: safety and efficacy evaluation in rhesus macaques. Cancer Res 72:3652-3663, 2012

18. McDannold N, Clement GT, Black P, Jolesz F, Hynynen K: Transcranial magnetic resonance imaging- guided focused ultrasound surgery of brain tumors: initial findings in 3 patients. Neurosurgery 66:323-332, 2010

19. McDannold N, Maier SE: Magnetic resonance acoustic radiation force imaging. Med Phys 35:3748-3758, 2008

20. McDannold N, Zhang YZ, Power C, Jolesz F, Vykhodtseva $\mathrm{N}$ : Nonthermal ablation with microbubble-enhanced focused ultrasound close to the optic tract without affecting nerve function. J Neurosurg 119:1208-1220, 2013

21. McDannold NJ, Vykhodtseva NI, Hynynen K: Microbubble contrast agent with focused ultrasound to create brain lesions at low power levels: MR imaging and histologic study in rabbits. Radiology 241:95-106, 2006

22. Miller DL, Gies RA: Enhancement of ultrasonically-induced hemolysis by perfluorocarbon-based compared to air-based echo-contrast agents. Ultrasound Med Biol 24:285-292, 1998

23. O'Reilly MA, Jones RM, Hynynen K: Three-dimensional transcranial ultrasound imaging of microbubble clouds using a sparse hemispherical array. IEEE Trans Biomed Eng 61:1285-1294, 2014
24. Pinton G, Aubry JF, Fink M, Tanter M: Numerical prediction of frequency dependent 3D maps of mechanical index thresholds in ultrasonic brain therapy. Med Phys 39:455-467, 2012

25. Radicke M, Engelbertz A, Habenstein B, Lewerenz M, Oehms O, Trautner P, et al: New image contrast method in magnetic resonance imaging via ultrasound. Hyperfine Interact 181:21-26, 2008

26. Sanghvi NT, Fry FJ, Foster RS, Bihrle R, Zaitsev A, Hennige $\mathrm{C}$ : High intensity focused ultrasound treatment of prostate tissue in the presence of an US contrast agent. J Ultrasound Med 14 Suppl:S17, 1995

27. Sassaroli E, Hynynen K: On the impact of vessel size on the threshold of bubble collapse. Appl Phys Lett 89:123901, 2006

28. Song J, Pulkkinen A, Huang Y, Hynynen K: Investigation of standing-wave formation in a human skull for a clinical prototype of a large-aperture, transcranial MR-guided focused ultrasound (MRgFUS) phased array: an experimental and simulation study. IEEE Trans Biomed Eng 59:435-444, 2012

29. Todorova M, Agache V, Mortazavi O, Chen B, Karshafian R, Hynynen K, et al: Antitumor effects of combining metronomic chemotherapy with the antivascular action of ultrasound stimulated microbubbles. Int J Cancer 132:2956-2966, 2013

30. Tran BC, Seo J, Hall TL, Fowlkes JB, Cain CA: Microbubble-enhanced cavitation for noninvasive ultrasound surgery. IEEE Trans Ultrason Ferroelectr Freq Control 50:12961304, 2003

31. Umemura S, Kawabata K, Hashiba K: Enhancement of ultrasonic absorption by microbubbles for therapeutic application, in 2001 IEEE Ultrasonics Symposium. Piscataway, NJ: IEEE, 2001, pp 1311-1314

32. Vykhodtseva N, McDannold N, Hynynen K: Induction of apoptosis in vivo in the rabbit brain with focused ultrasound and Optison. Ultrasound Med Biol 32:1923-1929, 2006

33. White PJ, Clement GT, Hynynen K: Longitudinal and shear mode ultrasound propagation in human skull bone. Ultrasound Med Biol 32:1085-1096, 2006

34. Xu Z, Carlson C, Snell J, Eames M, Hananel A, Lopes MB, et al: Intracranial inertial cavitation threshold and thermal ablation lesion creation using MRI-guided $220-\mathrm{kHz}$ focused ultrasound surgery: preclinical investigation. J Neurosurg 122:152-161, 2015

35. Yin X, Hynynen K: A numerical study of transcranial focused ultrasound beam propagation at low frequency. Phys Med Biol 50:1821-1836, 2005

36. Yu T, Wang G, Hu K, Ma P, Bai J, Wang Z: A microbubble agent improves the therapeutic efficiency of high intensity focused ultrasound: a rabbit kidney study. Urol Res 32:14-19, 2004

\section{Disclosure}

InSightec provided the TcMRgFUS system.

\section{Author Contributions}

Conception and design: Arvanitis, Vykhodtseva, McDannold. Acquisition of data: Arvanitis, Livingstone, McDannold. Analysis and interpretation of data: Arvanitis, Vykhodtseva, Livingstone, McDannold. Drafting the article: Arvanitis, Vykhodtseva, McDannold. Critically revising the article: Vykhodtseva, Jolesz, Livingstone, McDannold. Reviewed submitted version of manuscript: Jolesz, Livingstone, McDannold. Statistical analysis: Arvanitis, McDannold. Study supervision: McDannold.

\section{Correspondence}

Costas D. Arvanitis, Brigham and Women's Hospital, 221 Longwood Ave., Rm. 514a, Boston, MA 02115. email: cda@bwh. harvard.edu. 